\title{
Laser-induced narrowband coherent synchrotron radiation: Efficiency versus frequency and laser power
}

\author{
C. Evain, ${ }^{*}$ C. Szwaj, and S. Bielawski ${ }^{\dagger}$ \\ Laboratoire de Physique des Lasers, Atomes et Molécules, UMR CNRS 8523, \\ Centre d'Études et de Recherches Lasers et Applications, FR CNRS 2416, \\ Université des Sciences et Technologies de Lille, F-59655 Villeneuve d'Ascq Cedex, France
}

M. Hosaka and Y. Takashima

Graduate School of Engineering, Nagoya University, 464-8603 Nagoya, Japan

M. Shimada

UVSOR Facility, Institute for Molecular Science, National Institutes of Natural Sciences, Okazaki 444-8585, Japan

S. Kimura and M. Katoh

UVSOR Facility, Institute for Molecular Science, National Institutes of Natural Sciences, Okazaki 444-8585, Japan and School of Physical Sciences, The Graduate University for Advanced Studies (SOKENDAI), Okazaki 444-8585, Japan

\author{
A. Mochihashi \\ Japan Synchrotron Radiation Research Institute (JASRI), Spring-8, Sayo-cho, Hyogo 679-5198 Japan \\ T. Takahashi \\ Research Reactor Institute, Kyoto University, 590-049 Osaka, Japan
}

T. Hara

SPring-8/RIKEN, Sayo-cho, 679-5148 Hyogo, Japan

(Received 18 December 2009; published 27 September 2010)

\begin{abstract}
We analyze the narrowband terahertz emission process occurring from electron bunches passing in a bending magnet, after a laser-induced sinusoidal modulation has been performed. In particular, we focus on experimental tunability curves, and power scalings with current and laser power. Theoretically, we simplify the problem formulation using the slowly varying envelope approximation. At low powers, the scaling with laser power appears to be quadratic, and analytical expressions for the tuning curves are obtained. Emission at first passage in the bending magnet, and after one full turn in the storage ring, are considered both experimentally and theoretically. The experiments are performed on the UVSOR-II storage ring.
\end{abstract}

DOI: 10.1103/PhysRevSTAB.13.090703

PACS numbers: 42.65.Ky, 41.60.Ap, 41.75.Ht

\section{INTRODUCTION}

Interaction between a laser and a relativistic electron beam provides a way to manipulate the longitudinal charge distribution, and such experiments can lead to a coherent emission of terahertz radiation (coherent synchrotron radiation or CSR). The first experiments on laser-induced CSR have been realized in storage rings in the so-called conditions of slicing [1-3], where a short pulse interacts with a small part of the electron bunch. This leads to a

\footnotetext{
*Present address: Synchrotron SOLEIL, Saint Aubin, BP34, 91192 Gif-sur-Yvette, France.

${ }^{\dagger}$ serge.bielawski@univ-lille1.fr

*Present address: High Energy Accelerator Research Organization, KEK 305-0801, Tsukuba, Japan.
}

broadband terahertz emission at downstream bending magnets [3-6].

Recently, it has also been demonstrated that narrowband terahertz emission can also occur inside a bending magnet, when a sinusoidal modulation of the electrons has been induced by long laser pulse containing a sinusoidal amplitude modulation. After conjectures [4,7], recent experimental evidence was obtained in a storage ring, where the electron modulation was induced through interaction with a laser inside an undulator [8,9].

Then this strategy has also been considered in the IKNO project [10], and alternate modulation schemes related have also been reported. Experimentally, electron modulation at the photocathode of a photoinjector has been also realized [11]. Theoretical works by Xiang and Stupakov [12] on electron bunch modulation inside undulators have 
also revealed that emission on the high frequency side can also be obtained, using schemes involving two undulators.

In this paper we report a detailed study in the case where laser-electron interaction occurs inside a single undulator, in connection with our experiment at UVSOR-II. We focus, in particular, on the tunability of the emission, and on the scalings of power versus beam current and laser power. Experiments are made on the UVSOR-II storage ring, using a laser setup which is simplified with respect to the one of Ref. [8]. Theoretical calculations are made using a slowly varying envelope approximation, in a way similar to the work of Stupakov and Heifets [12,13]. Asymptotic expansions valid for long laser pulses and low power are found. They provide simple analytic formula for the estimation of the tuning range versus machine parameters, and also for the scaling of efficiency with laser power.

In a first step (Sec. II), we present the experimental dependence of terahertz emission power versus frequency, current, and laser power. Then, in Sec. IV we present selected theoretical and numerical results, the detailed theory being extensively presented in the Appendices.

\section{EXPERIMENTAL SETUP AND METHODS}

\section{A. Global organization of the experiment}

The experimental setup is represented in Figs. 1 and 2. The $800 \mathrm{~nm}$ laser pulses with a fast longitudinal intensity modulation (typically with a $1-10$ picosecond period and 300 ps FWHM duration) are sent on the electron bunch of the UVSOR-II storage ring (Fig. 1). The storage ring operates at $600 \mathrm{MeV}$, in single bunch mode, with currents in the $0-25 \mathrm{~mA}$ range, and the bunch duration is typically 100 ps RMS. The laser pulses are focused on the first undulator of the free-electron laser optical klystron. As for classical slicing experiments [1-3], the fundamental undulator frequency is tuned at the laser frequency.

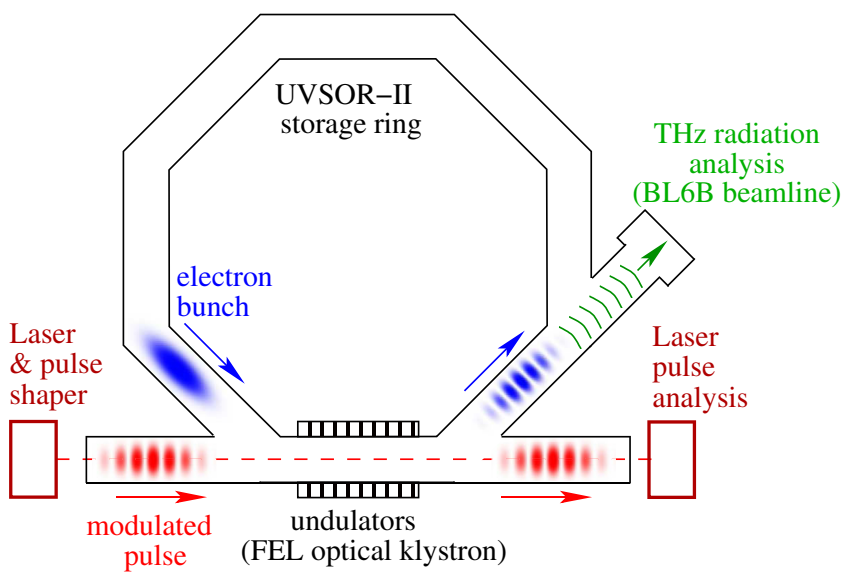

FIG. 1. (Color) Global view of the experiment at the UVSOR-II storage ring. Laser pulses with a sinusoidal modulation interact inside an undulator (FEL beam line), and the $\mathrm{THz}$ radiation analysis is performed at the terahertz BL6B beam line.

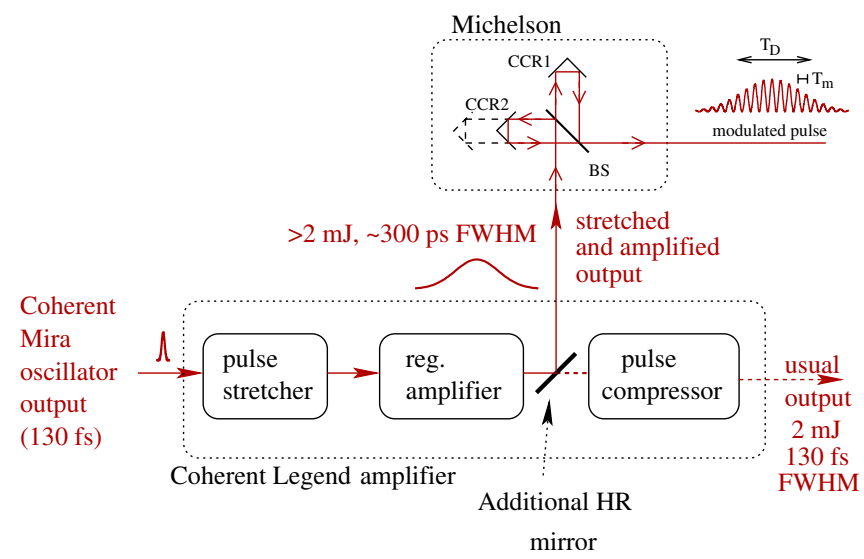

FIG. 2. (Color) Detail of the pulse shaping system. A regenerative chirped pulse amplifier (Coherent Legend) is slightly modified. A high-reflectivity mirror is placed between the amplifier and the compressor, to extract the high-energy stretched pulses. These latter are sent to a Michelson interferometer, and the resulting modulated pulses are then focused on the electron beam, as in usual slicing experiments. CCR1 and CCR2 are high precision corner cube hollow retroreflectors from PLX Inc. (OW-25-0563), i.e., with $63 \mathrm{~mm}$ total aperture, $<0.5$ arc sec deviation, and $\lambda / 4$ wavefront distortion (@633 nm).

Analysis of the terahertz emission is made at the BL6B beam line [14] (all $\mathrm{THz}$ paths occur in vacuum). Depending on experiments, we recorded directly the emitted power using an In-Sb hot-electron bolometer (QMC Instruments $\mathrm{QFI} / 2$, with $1 \mu$ s response time), or the $\mathrm{THz}$ spectrum, using a Martin-Puplett based Fourier-transform spectrometer (Jasco Faris 1).

For shaping sinusoidally the laser pulses, we have used a setup simpler than in Ref. [8]. The principle is still to use the so-called chirped pulse beating technique (see Ref. [15] for details), where a pulse experiences a strong dispersion before passing an interferometer (a Michelson interferometer here). However, instead of using a pair of gratings to achieve dispersion, we simply extract the $2 \mathrm{~mJ}$ pulse of our regenerative amplifier (Coherent Legend) just before the compressor, and send it to a Michelson interferometer (Fig. 2).

The shaped pulses were focused on the electron beam using a lens with $5 \mathrm{~m}$ focal length. Relative positions of the electron beam and the laser beam were monitored downstream, using a video camera. The camera lens $(2.5 \mathrm{~m}$ focal length) was placed at $9 \mathrm{~m}$ from the center of the first undulator.

\section{B. Modulated pulses: Shape expected theoretically from Weling-Auston theory}

The chirped pulse beating technique (CPB) has been extensively studied by Weling and Auston [15], and in this section we will essentially recall here their main results. We limit to the case where only second dispersion 
is considered, and leave consideration of third-order dispersion to further studies.

We assume that a Gaussian input pulse without chirp is present at the input of the compressor of Fig. 2:

$$
E_{L \mathrm{comp}}=E_{0} e^{-\left(t^{2} / 4 \Delta T_{L 0}^{2}\right)} e^{-i \omega_{0} t}
$$

with $\omega_{0}$ the pulsation of the optical carrier, and $\Delta T_{L 0}$ the RMS laser pulse duration.

If we assume a linear chirp at the output of the regenerative amplifier, the electric field can be written

$E_{\text {Lstreched }} \approx E_{0}\left(\frac{\Delta T_{L 0}}{\Delta T_{L}}\right)^{1 / 2} e^{-\left(t^{2} / 4 \Delta T_{L}^{2}\right)\left[1+i\left(\Delta T_{L} / \Delta T_{L 0}\right)\right]} e^{i\left(-\omega_{0} t+\phi_{0}\right)}$,

in the limit case $\Delta T_{L} \gg \Delta T_{L 0}$. This will be satisfied in our case where $\Delta T_{L}=125 \mathrm{ps}$ (i.e., $300 \mathrm{ps}$ FWHM) and $\Delta T_{L 0} \approx 55$ fs (i.e., $130 \mathrm{fs}$ FWHM). $\phi_{0}$ is a phase shift term which is not important in the present work.

The effect of the Michelson is to produce an interference between two delayed copies of this pulse. According to Ref. [15], the output intensity at the output of the system is

$$
\begin{aligned}
I_{\mathrm{out}}(t)= & I^{+}(t)+I^{-}(t)+E_{0}^{2}\left(\frac{\Delta T_{L 0}}{\Delta T_{L}}\right) \exp \left(-\frac{\tau^{2}}{8 \Delta T_{L}^{2}}\right) \\
& \times \exp \left(-2 \frac{t^{2}}{4 \Delta T_{L}^{2}}\right) \cos \left(2 \pi \nu_{m} t+\omega_{0} \tau\right),
\end{aligned}
$$

with $\tau$ the delay induced by the Michelson interferometer, and $I^{ \pm}(t)=\frac{1}{2} E_{0}^{2}\left[\left(\Delta T_{L 0}\right) /\left(\Delta T_{L}\right)\right] \exp \left[-(t \pm \tau / 2)^{2} / 2 \Delta T_{L}^{2}\right]$. The intensity is modulated at the frequency (in hertz)

$$
\nu_{m}=\frac{\tau}{4 \pi \Delta T_{L 0} \Delta T_{L}}
$$

This may also be expressed as

$$
\nu_{m}=\Delta \nu_{L}\left(\frac{\tau}{\Delta T_{L}}\right)
$$

where $\Delta \nu_{L}$ is the RMS spectral width of the laser $\left(2 \pi \Delta \nu_{L} \Delta T_{L 0}=1 / 2\right)$. In Eq. (5), we can see clearly that the modulation frequency is linearly proportional to the Michelson delay $\tau$.

\section{Expected tunability limits}

\section{Limits of the laser pulse shaper}

To reach high frequencies, large delays are needed, and the main limitation will come from the necessity to keep an overlap between the pulses at the Michelson's output. This corresponds to the attenuation term $\exp \left(-\frac{\tau^{2}}{8 \Delta T_{L}^{2}}\right)$ in Eq. (3).
Maximum frequency is there expected for $\tau$ of the order of $\Delta T_{L}$. Using Eq. (5), it is easily shown that the maximum value for the modulation frequency $\nu_{m}$ is limited by the laser spectral width $\Delta \nu_{L}$.

Here, the FWHM spectral width is typically of the order of $11 \mathrm{~nm}$ and $\Delta T_{L} \approx 125 \mathrm{ps}$, thus the available modulation frequencies are from $\approx 100 \mathrm{GHz}$ to several tens of $\mathrm{THz}$. We will see in the following that this will not put a strong limit, as beam transport will provide the main upper frequency limitation in our experiment.

An additional expected limitation comes from thirdorder dispersion effects [15]. Here we will not enter this question in detail, and leave the related topics (influence on terahertz emission and attempts to reduce third-order dispersion) to further studies.

\section{Remark on the limits linked to undulator or optical klystron parameters}

Let us note also that, in general, reaching high frequencies may require large laser bandwidth, and this may lead ultimately to consequences on undulator (or optical klystron) parameters. More precisely, for the laser-electron interaction to be actually efficient, the laser spectrum should be less than the undulator (or optical klystron) optical bandwidth $\Delta \nu_{u}$.

This is not a strong limit in our case because the laser is focused on the first undulator. Indeed, we expect the bandwidth acceptance of the undulator to be of the order of $\Delta \nu_{U}=c /(N \lambda)$, with $\lambda$ the laser wavelength and $N$ the number of undulator periods. This is an order of magnitude larger than the laser spectral width.

This linewidth criterion may be also expressed in time domain. We may expect that the undulator (or optical klystron) spectral width would not present a strong limit, as long as the slippage length is smaller than $c / \Delta \nu_{L}$.

\section{Modulated pulses: Experimental characterization methods}

We characterized the output pulses using an autocorrelator, an optical spectrum analyzer, and a streak camera (Hamamatsu C5680). Recorded data were consistent with the expected results from Weling and Auston.

At very low wave numbers, the resolution of the streak camera ( $2 \mathrm{ps})$ allowed direct recording of pulse shapes. The modulation was clearly visible and presented a satisfying contrast (Fig. 3). Its period could be varied as expected, by changing the delay on the Michelson interferometer. Direct streak camera recording provided also information on real laser pulse shape (with and without pulse shaper), and also on residual phase fluctuations of the modulation. For instance, we can deduce from Fig. 3(a) that mechanical fluctuations of Michelson mirror positions of the order of hundreds of nanometers are present.

At higher wave numbers (typically $2-30 \mathrm{~cm}^{-1}$ ), where main experiments were done, streak camera resolution was 

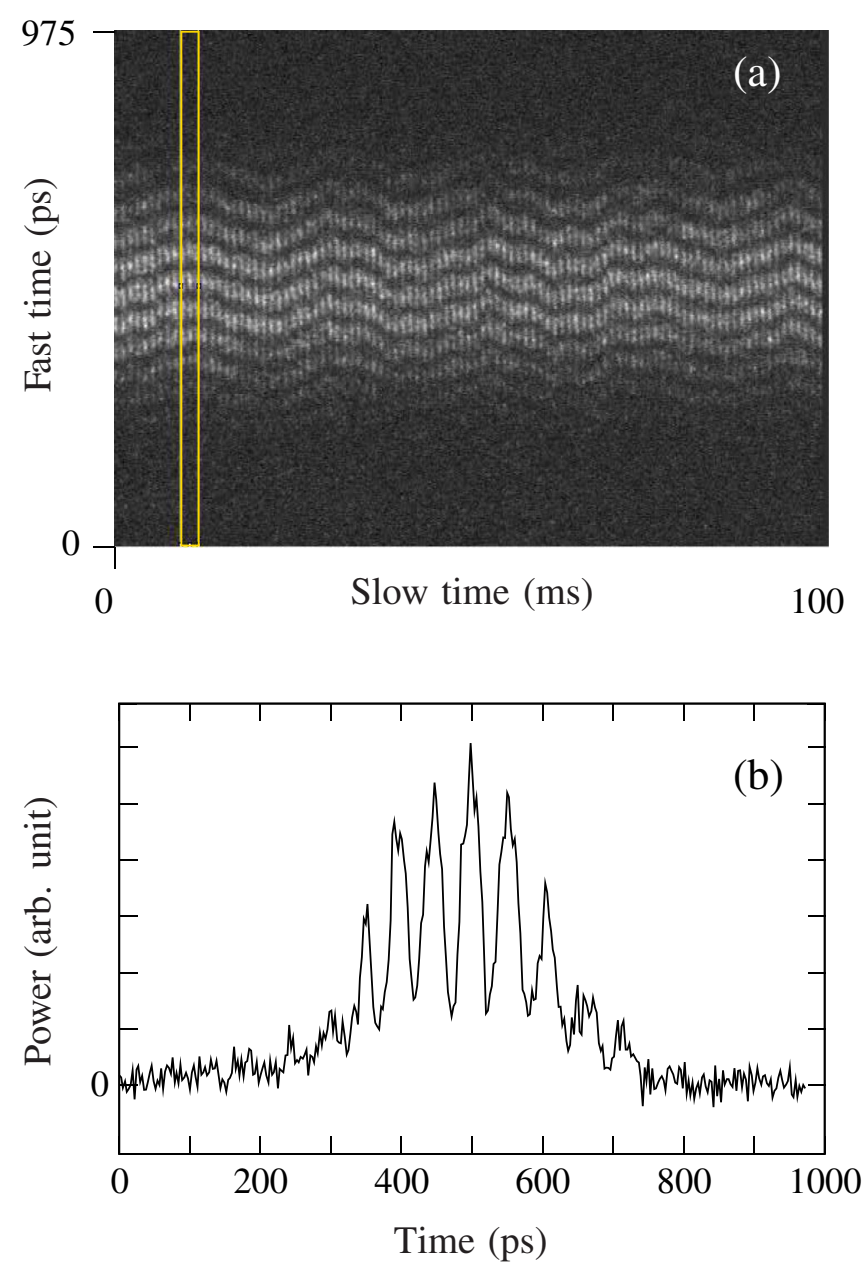

FIG. 3. (Color) Pulse shape, checked at very low modulation wave number, using a double sweep streak camera. (a) Raw streak camera image, where 100 successive pulses are recorded. (b) Pulse shape averaged over $3 \mathrm{~ms}$, i.e., the rectangular area displayed in (a). Note that the phase fluctuations in (a) are due to mechanical vibrations of the Michelson's mirrors (essentially at $60 \mathrm{~Hz}$ ), with an amplitude of several hundreds of nanometer.

not sufficient, and routine checks of the pulse modulation period were obtained from autocorrelation measurements. Preliminary experiments with short duration pulse, which were made using an external pulse stretcher [8], were characterized by a collinear two-photon autocorrelator, using a GaAsP photodiode [16,17]. Typical autocorrelations are presented in Fig. 4(a), and the corresponding optical spectrum in Fig. 4(b). With the new pulse shaping system presented in Fig. 2, we built a long range noncollinear autocorrelator based on a beta barium borate (BBO) second-harmonic generation (SHG) crystal, and a synchronous recording of the $\mathrm{SHG}$ pulses (at $1 \mathrm{KHz}$ ). A typical autocorrelation curve is presented in Fig. 4(c). The autocorrelation system was used to check the consistency between the pulse modulation and the terahertz emission frequency, in particular, in the preliminary phase of the experimental investigations.
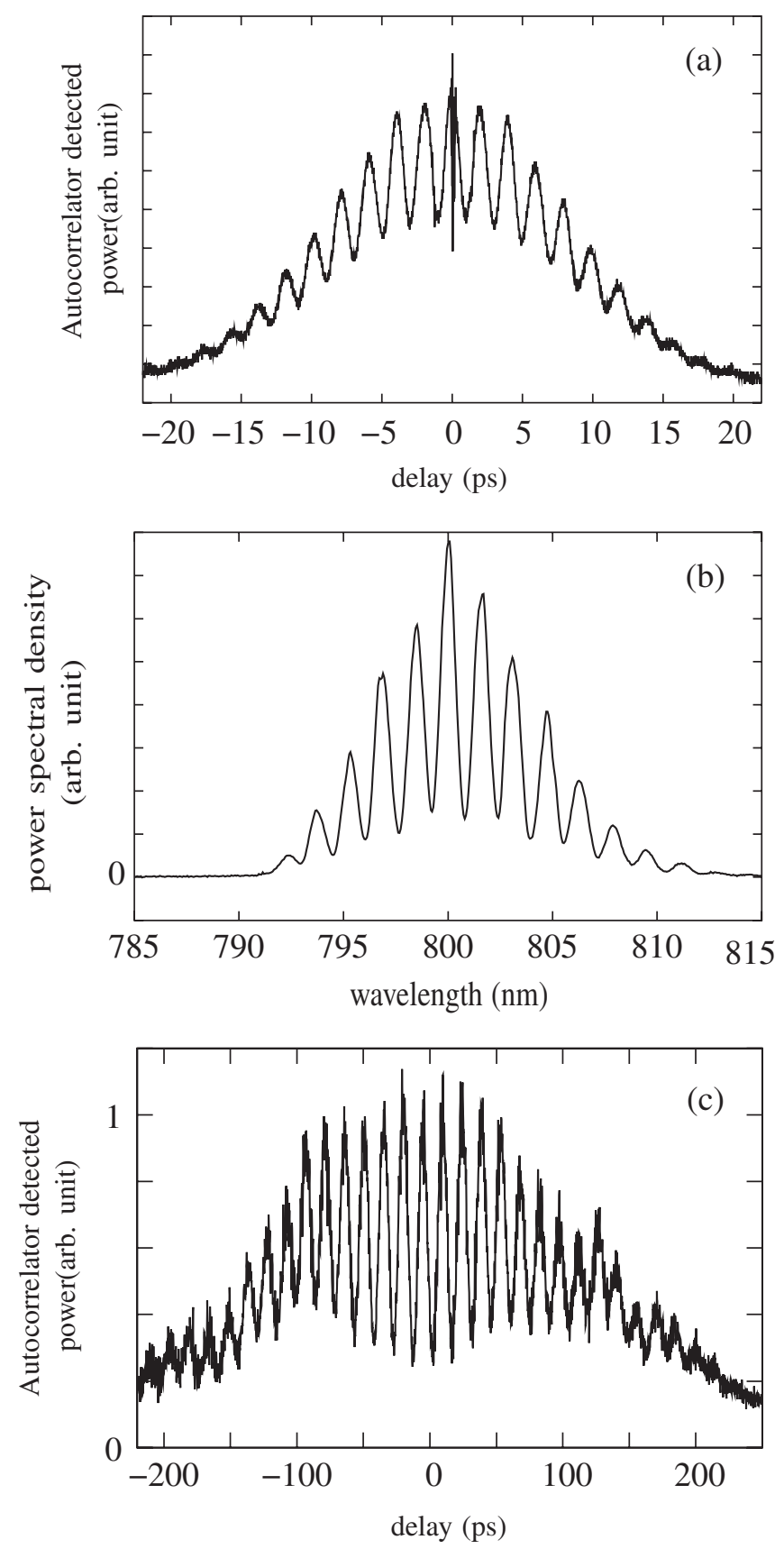

FIG. 4. Typical autocorrelation trace (a) and spectrum (b) of the relatively short pulses (in the 10 ps range) obtained with the previous setup, which used an external pulse stretcher. (c) Autocorrelation of long pulses ( $\approx 300$ ps FWHM) using the actual pulse shaper.

\section{EXPERIMENTAL RESULTS}

\section{A. Tunability range, wavelength dependence of the efficiency}

The obtained pulses have a duration of $300 \mathrm{ps,} \mathrm{and}$ their internal modulation frequency can be changed at will, simply by adjusting the position of one of the Michelson's retroreflector positions. Typical coherent ter- 


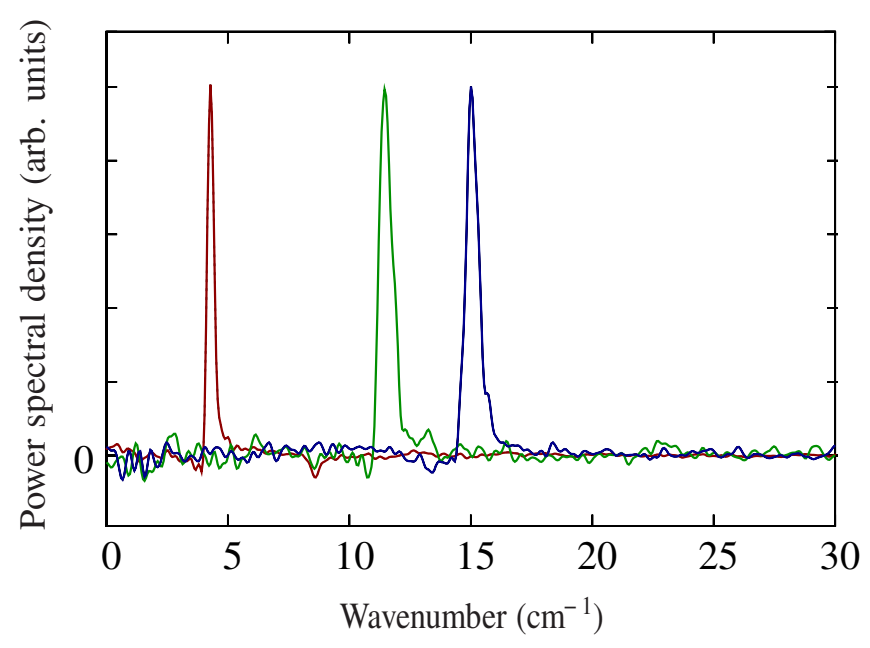

FIG. 5. (Color) Three typical emission spectra induced by the shaped laser pulses, for three different modulation frequencies. Each spectrum is rescaled to its maximum value.

ahertz emission spectra are represented in Fig. 5. As in Ref. [8], we observe a linear dependence of the peak emission frequency with respect to the Michelson mirror position (Fig. 6). The major difference with Ref. [8] concerns the tuning range, which appears now much wider. A detectable signal is now observed over more than one decade. This may be a priori attributed to the larger pulse duration used here (300 ps versus 50 ps in Ref. [8]), which allows one to excite modulations at lower frequencies.

To investigate in more detail the frequency dependence of the efficiency, we have realized experiments where we record the signal detected by the bolometer versus the excitation frequency. Observation of the raw data [Fig. 7(a)] already suggests that efficient emission occurs at two characteristic frequency domains. However, this curve is not linked to the frequency dependence of the emitted power in a simple way, because various uncon-

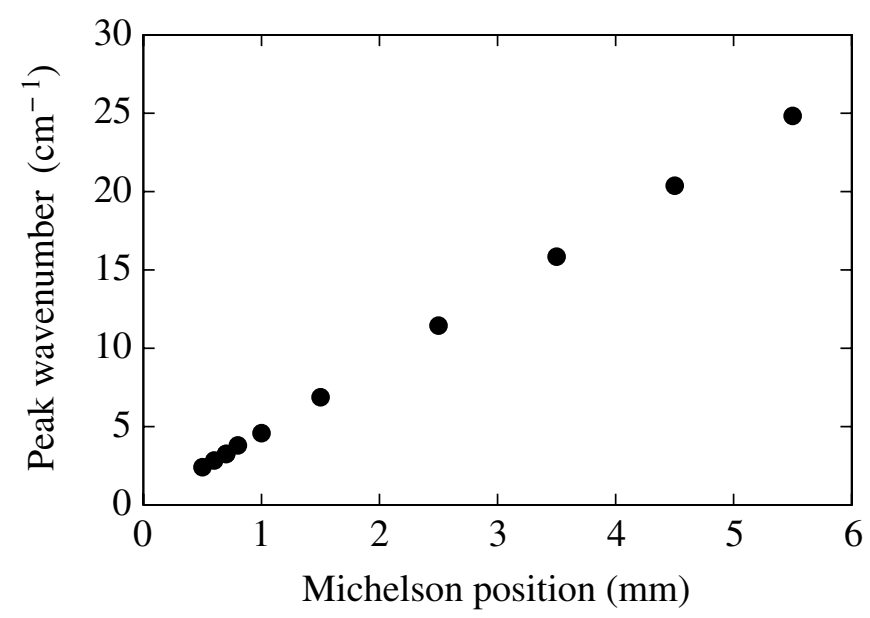

FIG. 6. Dependence of the peak terahertz emission frequency with the position of the Michelson mirror.
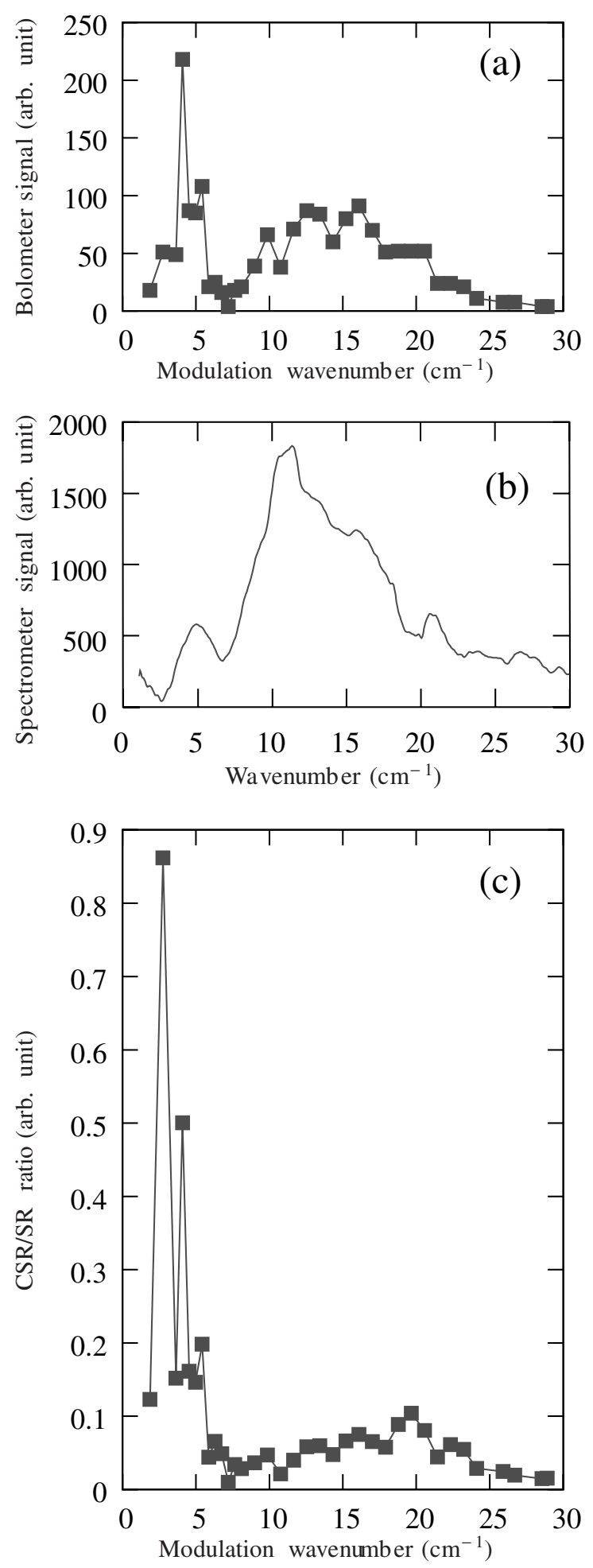

FIG. 7. Response versus modulation wave number. (a) Signal detected by the bolometer versus modulation wave number (note that the shape is affected by the frequency responses of the bolometer, the beam line, etc.). (b) Reference spontaneous SR spectrum without laser. (c) Ratio between CSR and spontaneous SR [curve (a)/curve (b)]. In (c), the vertical scale is arbitrary. The typical values will be measured in Sec. III C. At $15 \mathrm{~cm}^{-1}$, when the current is $10 \mathrm{~mA}$, the typical pulse energy ratio is of the order of 20000 . 
trolled frequency responses (beam line, windows, etc.) are involved in the process.

Another way to represent the data consists of dividing this frequency response by the spontaneous synchrotron radiation spectrum [Fig. 7(b)], i.e., without laser. This will allow one to compare the experimental results with theory, which will provide the frequency dependence of the form factor. The results are presented in Fig. 7(c).

We will see in the theoretical part that the broad $20 \mathrm{~cm}^{-1}$ peak is due to the CSR emission occurring after a straight transport from the undulator to the bending magnet of the $\mathrm{THz}$ beam line. The low frequency emission (around 3-5 $\mathrm{cm}^{-1}$ ) is attributed to the emission after long transport in the storage ring (typically one full turn or more). Although this experiment does not provide absolute values of the powers nor power ratios (quantitative measurements will be the purpose of Sec. III C), the particular shape of this curve already provide reference data against which theory will be compared.

\section{B. Efficiency versus laser power and beam current}

In these experiments, we recorded the dependence of the emitted power with current and with laser power. We attenuated the laser using a half-wave plate (HWP) followed by a polarizer, and we paid particular attention to ensure that the laser beam location was not moving when the HWP was rotated. This was ensured by the choice of a low-angular error HWP (CVI QWPO-800-10-2-R15, with a parallelism error lower than 0.5 arcsec).

Dependence with current appeared quadratic with a good approximation [Fig. 8(a)], as expected from CSR processes in general [18-20]. By rotating the half-wave plate, we obtained the dependence of terahertz power with incident laser power [Fig. 8(b)]. This dependence also appears to be quadratic with a good approximation. We will see in the theoretical section that this quadratic scaling is generally expected in the limit of low laser powers (independently of the laser pulse shape).

\section{Comparative study of CSR and spontaneous synchrotron radiation power densities}

Over the bandwidth of interest, the energy per pulse delivered by the CSR process is larger than the one delivered by the "normal" spontaneous synchrotron radiation. To evaluate in a quantitative way the ratio between the efficiencies of the two processes, we recorded power spectra with the laser ON (CSR) and with laser OFF (normal synchrotron radiation). The excitation wave number was $15 \mathrm{~cm}^{-1}$.

From these data, we deduced the ratio

$$
\eta_{\mathrm{CSR} / \mathrm{SR}}(\bar{\nu})=\frac{Q_{\mathrm{CSR}}(\bar{\nu})}{Q_{\mathrm{SR}}(\bar{\nu})},
$$

where $\bar{\nu}$ is the spectroscopic wave number, $Q_{\mathrm{CSR}}(\bar{\nu})$ is the energy spectral density of a single CSR pulse, and $Q_{\mathrm{SR}}(\bar{\nu})$
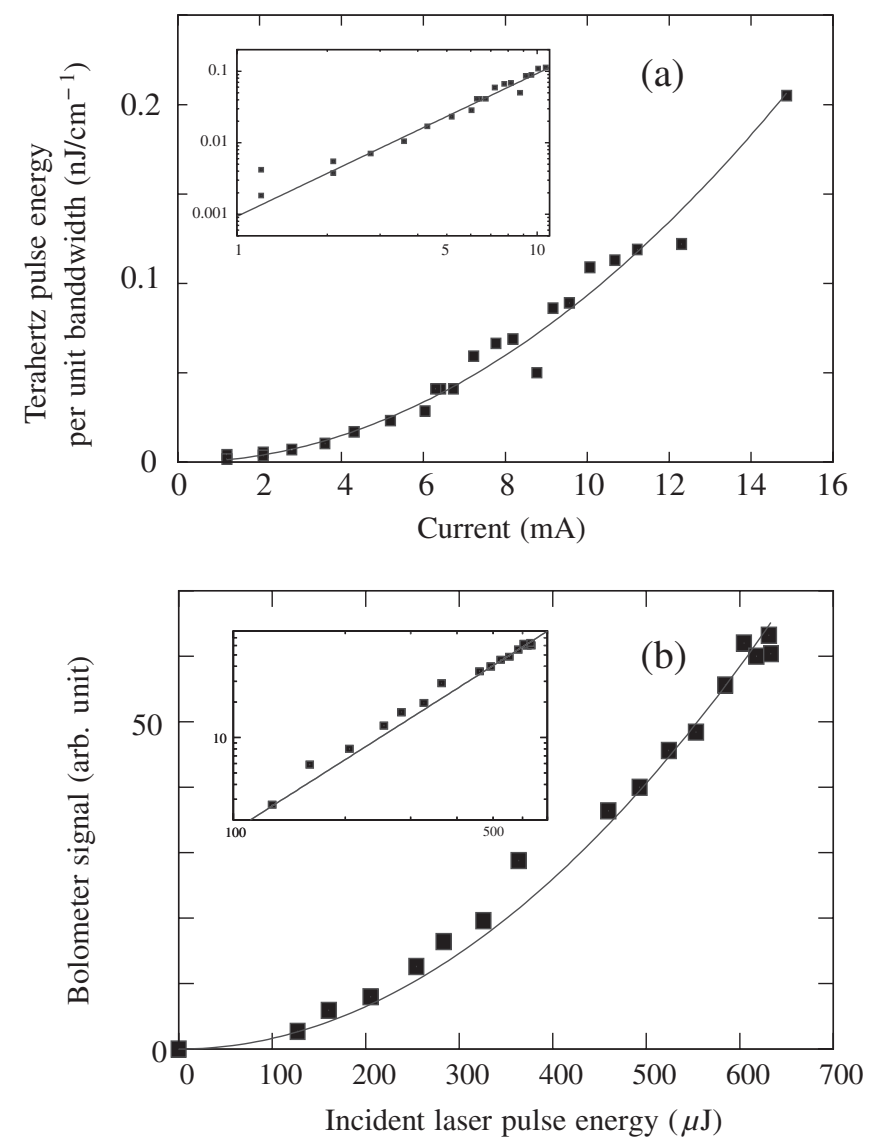

FIG. 8. Terahertz energy per bandwidth unit versus beam current (a), and versus laser pulse energy (b). Squares: experimental data; full line: quadratic law fit. The insets represent the same curves in log-log units. In (a), the laser pulse energy is $800 \mu \mathrm{J}$. In (b), the current is $4.8 \mathrm{~mA}$. The modulation wave number is $15 \mathrm{~cm}^{-1}$.

is the energy spectral density the normal SR emitted by a single unmodulated bunch. The ratio $\eta_{\mathrm{CSR} / \mathrm{SR}}\left(15 \mathrm{~cm}^{-1}\right)$, plotted in Fig. 9, reveals that CSR terahertz pulse energy in the bandwidth of interest was larger by a factor $10^{3}-10^{4}$ with respect to normal SR.

An estimation of the form factor can be obtained from the $\eta_{\mathrm{CSR} / \mathrm{SR}}(\bar{\nu})$ ratio. For the sake of simplicity, let us assume that diffraction properties are identical for SR and CSR. In this case, according to Refs. [20,21], the ratio is

$$
\eta_{\mathrm{CSR} / \mathrm{SR}}(\bar{\nu})=\frac{Q_{\mathrm{CSR}}(\bar{\nu})}{Q_{\mathrm{SR}}(\bar{\nu})}=(N-1) f(\bar{\nu}),
$$

with $N$ the number of electrons in the bunch. $f(\bar{\nu})$ is the socalled form factor.

In the conditions of Fig. 9, the ratio is $\approx 2000$ per $\mathrm{mA}$ for a laser pulse energy of $800 \mu \mathrm{J}$. Since the number of electrons in the bunch is $0.11 \times 10^{10}$ per $\mathrm{mA}$, and the terahertz power scales quadratically with laser power, the form factor is estimated to be 


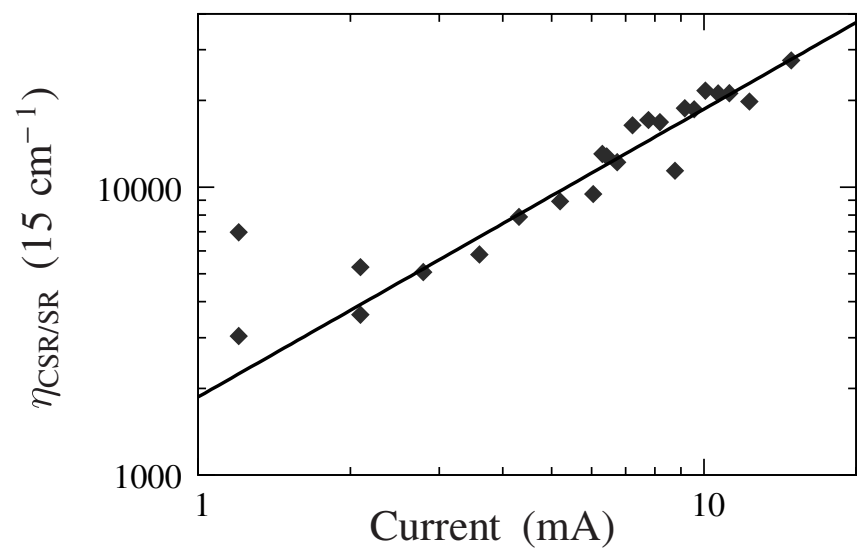

FIG. 9. Ratio between CSR and incoherent SR terahertz energy per pulse and unit bandwidth, at the modulation frequency $\left(15 \mathrm{~cm}^{-1}\right)$. Squares: experimental data; full line: linear fit. Incident laser pulse energy: $800 \mu \mathrm{J}$.

$$
\left|\tilde{\rho}\left(15 \mathrm{~cm}^{-1}\right)\right|^{2}=3 \times 10^{-6} Q_{L}^{2}
$$

at the excitation frequency, with $Q_{L}$ the laser pulse energy (in $\mathrm{mJ}$ ).

This type of experiment can also provide quantitative estimation of the CSR pulse energy per unit bandwidth emitted in the bending magnet, from the knowledge of the SR power emitted theoretically by one electron. In our case the CSR energy per pulse is thus estimated to be $0.1 \mathrm{~nJ}$, when the beam current is $10 \mathrm{~mA}$.

These values are expected to change by large factors when different experimental conditions will be considered. For instance, concerning specifically laser pulse parameters, in the next experiments at UVSOR we plan to use a higher energy laser, and we plan to use shorter pulses (using an adjustable compressor). For instance, increasing pulse energy by a factor $25(50 \mathrm{~mJ}$ instead of $2 \mathrm{~mJ}$ here $)$ would allow one to increase the terahertz peak power by a factor of the order of $(50 / 2)^{2}=625$ (assuming a quadratic dependence of terahertz power versus laser power). Decreasing the pulse duration $\tau_{L}$ is expected also to allow one to increase the $\mathrm{THz}$ pulse peak power in the present conditions, and is also a parameter to be optimized. Dependence on machine parameters (energy, beam line magnet, etc.) is expected to be more subtle [9,22], and further calculations and experiments would be worth trying on other machines, case by case.

\section{THEORETICAL AND NUMERICAL RESULTS}

In this section, we study numerically and analytically the dependence of the terahertz emission properties versus selected frequency, and versus laser power. As in previous works [4,23], we focus essentially on the calculation of the so-called form factor $f(\bar{\nu})$, introduced in Eq. (7), which measures the ratio between CSR and SR energy density spectra.

The wave number $\bar{\nu}$ in Eq. (7) will be expressed in reduced units in the Appendices' calculation. However, the values reached by the form factor [Eq. (7)] does not depend on the choice of the wave number unit. Note that in the Appendices, the form factor will be expressed as

$$
f(\bar{\nu})=|\tilde{\rho}(k)|^{2},
$$

where $\tilde{\rho}(k)$ is the Fourier transform of the longitudinal charge density distribution. The dimensionless wave number $k$, which will be used throughout the Appendices, is related to the spectroscopic wave number $\bar{\nu}\left(\right.$ in $^{-1}$ ) as follows:

$$
k=2 \pi \bar{\nu} \times \sigma_{z},
$$

with $\sigma_{z}$ the RMS bunch length.

Calculation of the form factor $|\tilde{\rho}(k)|^{2}$ implies the calculation of an integral with rapidly varying integrand, at the optical and $\mathrm{THz}$ frequencies. As a key point, we take advantage of this two different scales (the envelope of a laser pulse varies typically much slowly that the optical carrier) and apply the slowly varying envelope approximation. This allows one to perform a drastic simplification of the problem. The details are given in the Appendices and, in this section, we limit ourselves to present the results which can be directly compared to our experimental data. The first result concerns the derivation of the form factor [Eq. (A13)] valid for any pulse shape and power. This provides a way to estimate numerically the spectrum of terahertz emission, with very short calculation times.

\section{A. Analytical expression for the peak emission frequency (in the limit of low laser power)}

Simple analytical formulas for the tuning range and emission spectra can also be obtained in the limit of low laser power. We present results in the limit of long laser pulses with a sine modulation (infinitely long laser pulses, and finite-size Gaussian pulses).

In the limits of low laser power, and assuming a plane wave for the laser and an infinitely long laser pulse and electron bunch (see Appendices B and C for details), the emitted CSR power is found to obey the following dependence (in the $\mathrm{THz}$ domain):

$$
\begin{aligned}
P_{\mathrm{CSR}}(\nu) / P_{\mathrm{SR}}(\nu) \approx & C P^{2}\left(R_{56} \sigma_{\delta}\right)^{4}(2 \pi \nu / c)^{4} \\
& \times e^{-\left(R_{51}^{2} \sigma_{x}^{2}+R_{52}^{2} \sigma_{x^{\prime}}^{2}+R_{56}^{2} \sigma_{\delta}^{2}\right)(2 \pi \nu / c)^{2}} .
\end{aligned}
$$

In this relation, $\nu$ is the modulation frequency of the laser pulse envelope (in hertz), and $P$ is the laser power. $R_{51}, R_{52}$, $R_{56}$ are the elements of the magnetic transport matrix from the laser-electrons interaction to the region of terahertz emission. $\sigma_{x}, \sigma_{x}^{\prime}$ are the electron bunch dimensions in 
phase space along the $x, x^{\prime}$, and $\sigma_{\delta}$ the relative energy spread. $c$ is the velocity of light. $C$ is a parameter, which we will not try to detail at the present stage of the modeling. $C$ is proportional to the square of the current, and depends on the details of the undulator.

We deduce that the dependence of terahertz power is quadratic with the incident laser power, as for the process of optical rectification in classical nonlinear optics [24,25]. The dependence of $P_{\mathrm{THz}}$ with frequency is a single bellshaped curve, centered at

$$
\nu_{\mathrm{mopt}}=\frac{c}{2 \pi} \frac{\sqrt{2}}{\sqrt{R_{51}^{2} \sigma_{x}^{2}+R_{52}^{2} \sigma_{x^{\prime}}^{2}+R_{56}^{2} \sigma_{\delta}^{2}}} .
$$

This expression provides a simple way to evaluate the possible terahertz emission frequency range in a project of laser-induced CSR terahertz production, provided the magnetic transport parameters and electron bunch parameters are known [for instance Eq. (12) gives the correct emission peak frequency of $0.6 \mathrm{THz}$ (i.e., $20 \mathrm{~cm}^{-1}$ )]. A similar expression including the Gaussian dependence of the laser pulse envelope is also given in Appendix B, and more generally the calculation can be modified easily to take into account other laser shapes.

\section{B. Numerical calculation of the emitted spectrum shape in the present experimental conditions}

In Fig. 10(a), we have represented the dependency of the form factor versus modulation wave number, using the UVSOR-II parameters of our experiments $\left(R_{i j}\right.$ coefficients and beam parameters). The less well-known parameter concerns the amplitude of the laser-induced energy modulation. However. this does not noticeably affect the curve shapes in the limit of low powers.

In the numerical work, the bunch parameters used are (inside the undulator): $\sigma_{x}=4.17 \times 10^{-4} \mathrm{~m}, \sigma_{x^{\prime}}=4.2 \times$ $10^{-5}, \sigma_{z}=3.1 \times 10^{-2} \mathrm{~m}, \sigma_{\delta}=3.4 \times 10^{-4}$.

The transport matrix elements $R_{i j}$ are displayed in Table I.

Using $R_{i j}$ coefficients corresponding to a straight transport from the undulator to the beam line's bending magnet, we find that the form factor has a peak at $20 \mathrm{~cm}^{-1}$ [curve labeled "turn 0" in Fig. 10(a)], in quantitative agreement with experimental findings [Fig. 7(c)]. CSR emission after one or more turns in the storage ring occurs at smaller frequencies. To compare the numerical results with the experimental ones, we have summed the contributions of turn 0, 1, etc. [Fig. 10(b)], because the bolometer speed does not allow one to resolve the successive $\mathrm{THz}$ pulses. These calculations do not take the diffusion of the structure, in particular, due to synchrotron radiation. However, the presence of the low frequency peak is in semiquantitative agreement by the $3-5 \mathrm{~cm}^{-1}$ peak observed experimentally in Fig. 7(c).
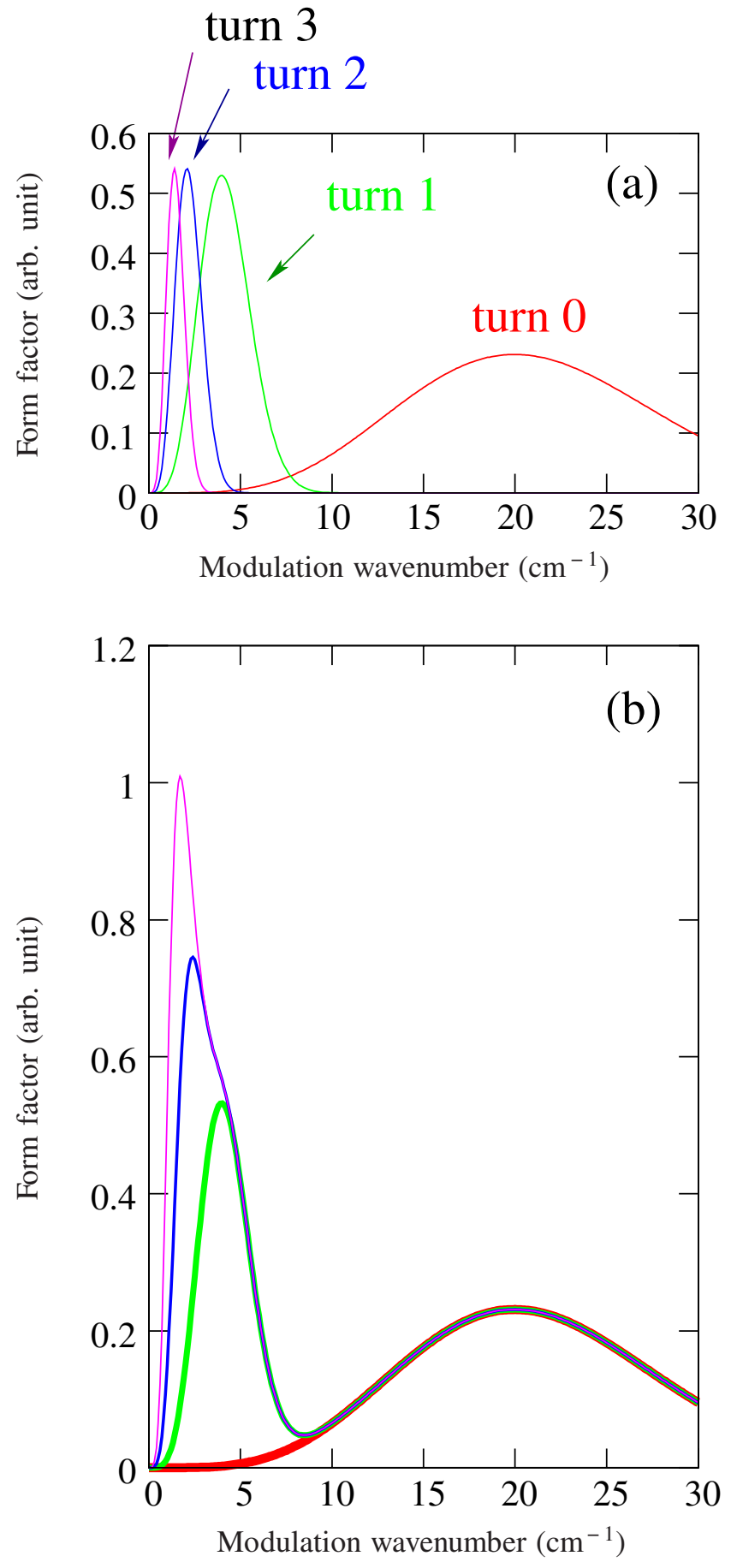

FIG. 10. (Color) (a) Dependence of the form factor versus wave number, using Eqs. (11) and (B3). The vertical unit is arbitrary in the sense that we took $P=C=1$ in the calculation. "turn 0 " corresponds to the parameters $R_{i j}$ associated with a straight transport from the undulator to the bending magnet. Labels "turn 0", "turn 1", "turn 2", "turn 3", correspond to $R_{i j}$ parameters associated to a straight transport, 1 full turn, 2 full turns, 3 full turns in the storage ring, respectively. Part (b) represents the sum of the first form factors: turn 0 only (red), sum of turns 0 and 1 (green), sum of turns 0,1 , and 2 (blue), sum of turns $0,1,2$, and 3 (magenta). 
TABLE I. Transport matrix parameters corresponding to different transport paths from the undulator to the $\mathrm{THz}$ beam line bending magnet: Direct transport, transport involving one full turn in the storage ring, 2 turns, etc.

\begin{tabular}{lclll}
\hline \hline & Straight transport & 1 full turn & 2 full turns & 3 full turns \\
\hline$R_{51}$ & 0.0396 & -0.073 & -0.0388 & 0.0732 \\
$R_{52}$ & $-1.53 \mathrm{~m}$ & $-1.19 \mathrm{~m}$ & -0.040 & -0.401 \\
$R_{56}$ & $-0.268 \mathrm{~m}$ & $-1.66 \mathrm{~m}$ & -3.19 & -4.77 \\
\hline \hline
\end{tabular}

\section{CONCLUSION}

From the theoretical point of view, the tuning range of the laser-induced narrowband CSR can be estimated theoretically using a simple approach. More precisely, in the plane wave approximation, the Fourier transform of the longitudinal charge density distribution can be calculated numerically using a simple quadrature, for any laser longitudinal shape. Moreover, maximum emission frequency can be estimated using analytic expressions. It is also important to notice that the approach can also be used in slightly different contexts. In particular, Eq. (A13) is valid for any laser pulse shape; it may be used for studies of conventional slicing (with nonmodulated pulses).

Important future steps in the theoretical and experimental investigations will concern the quantitative calculations of the power, and also a detailed study of the diffraction process occurring during emission [26,27]. More generally, the question of fundamental limits for the achievable power in these conditions remains an open problem.

Another important point concerns the effect of collective effects, due to wakefields (either from chamber shielding and bending magnets), and which were neglected in the theory. In the present experiments, we did not find experimental disagreements with theory. However, we think that further investigations using laser-induced electron bunch perturbations should be also made in different conditions, for which collective effects are more likely to have an effect. In particular, a possibility would be to observe the response over several turns in the storage ring, at high current, for instance near the threshold for CSR instability, as, e.g., for Ref. [28]. We think that this type of experiment may thus provide new informations on machine and CSR wakefields.

\section{ACKNOWLEDGMENTS}

We would like to thank M. E. Couprie from Synchrotron SOLEIL for key interactions and fruitful discussions. This work was supported by the Joint Studies Program (20052007) of the Institute for Molecular Science, and the JSPS fellowship program for research in Japan (Grant No. S06215). Part of this work was supported by Grantin-aid for scientific researches (B20360041) of JSPS. The CERLA is supported by the French Ministère chargé de la
Recherche, the Région Nord-Pas de Calais and the FEDER.

\section{APPENDIX A: DERIVATION OF SPECTRUM EXPRESSIONS VALID FOR ARBITRARY LASER PULSE SHAPES AND POWER}

\section{Formulation of the problem}

From a theoretical point of view, the wavelength range can be estimated from a simple approach [4,23]. In this process, the relevant spatial variables for an electron are its longitudinal coordinate $z$ and transverse coordinate $x$ in the horizontal plane, as well as the transverse velocity $x^{\prime}$ and relative energy shift $w$ from the center energy of the bunch.

Before interaction, the charge density distribution is taken Gaussian:

$$
f\left(x, x^{\prime}, z, w\right)=\frac{1}{4 \pi^{2}} e^{-(1 / 2)\left(x^{2}+x^{\prime 2}+z^{2}+w^{2}\right)},
$$

where all variables $\left(x, x^{\prime}, z, w\right)$ are expressed in units of their RMS values $\sigma_{x}, \sigma_{x^{\prime}}, \sigma_{z}, \sigma_{\delta}$.

The modeling of the process is simplified by considering two separate steps: (i) an energy modulation of the electrons by the laser, and (ii) the electron beam transport through dipole magnets. Interaction with the laser leads mainly to a fast energy modulation, at the optical wavelength scale, of the electron charge distribution. Assuming the simplified case of a plane wave for the laser, resulting in a sinusoidal modulation of the energy distribution, the phase-space distribution becomes

$$
g\left(x, x^{\prime}, z, w\right)=\frac{1}{4 \pi^{2}} e^{-(1 / 2)\left(x^{2}+x^{\prime 2}+z^{2}\right)} e^{-(1 / 2)[w-a(z) \sin (z / \epsilon)]^{2}},
$$

with $a(z)$ the laser field envelope, and $1 / \epsilon$ the laser wavelength in dimensionless units. In the units used here, $|a(z)|$ is the amplitude of the energy modulation in energy spread units.

Then the beam transport through the magnetic fields leads to path lengths which depend on energy, and also on $x$ and $x^{\prime}$. This is the main process which will transform the fast-scale energy modulation into a slow charge density modulation at the $\mathrm{THz}$ scale. Here we assume that this leads to a linear transformation of the coordinates $\left(x, x^{\prime}, z, w\right)=\mathbf{x}$ of each electron:

$$
\mathbf{x} \rightarrow \mathbf{r x},
$$

with $\mathbf{r}$ the normalized transport matrix [4] for the process. We assume that it has the following structure:

$$
\mathbf{r}=\left(\begin{array}{cccc}
r_{11} & r_{12} & 0 & r_{16} \\
r_{21} & r_{22} & 0 & r_{26} \\
r_{51} & r_{52} & 1 & r_{56} \\
0 & 0 & 0 & 1
\end{array}\right)
$$

The $r_{i j}$ coefficients are normalized as follows (only the 
useful terms $r_{i j}$ are shown):

$$
r_{51}=R_{51} \frac{\sigma_{x}}{\sigma_{z}}, \quad r_{52}=R_{52} \frac{\sigma_{x}^{\prime}}{\sigma_{z}}, \quad r_{56}=R_{56} \frac{\sigma_{\delta}}{\sigma_{z}},
$$

with $R_{i j}$ the usual unnormalized transport matrix elements [23]. The electron distribution is transformed into [4,23]

$$
h(\mathbf{x})=|\mathbf{r}|^{-1} g\left(\mathbf{r}^{-1} \mathbf{x}\right) .
$$

Then, to get information on the radiated $\mathrm{THz}$ field, it is important to examine the longitudinal charge density distribution, which acts as a source in the process [4]:

$$
\rho(z)=\int_{-\infty}^{+\infty} \int_{-\infty}^{+\infty} \int_{-\infty}^{+\infty} h\left(x, x^{\prime}, z, w\right) d x d x^{\prime} d w
$$

and, in particular, its Fourier transform:

$$
\tilde{\rho}(k)=\int_{-\infty}^{+\infty} e^{-i k z} \rho(z) d z
$$

Since $|\tilde{\rho}(k)|^{2}$ is precisely the form factor [21]. After substituting Eqs. (A6) and (A7) in Eq. (A8), quadratures over $x, x^{\prime}$, and $w$ can be easily performed using a change of variables (see Appendix D for details):

$$
\tilde{\rho}(k)=e^{-(1 / 2)\left(r_{51}^{2}+r_{52}^{2}+r_{56}^{2}\right) k^{2}} \psi(k)
$$

with $\quad \psi(k)=\int_{-\infty}^{+\infty} e^{-i k z} \frac{e^{-\left(z^{2} / 2\right)}}{\sqrt{2 \pi}} e^{i k r_{56} a(z) \sin (z / \epsilon)} d z$

This basic expressions for the obtained bunch shape and its spectrum, made in Eqs. (A7)-(A9), though important in the modeling, is difficult to integrate directly numerically. Indeed, the integrand presents variations at very different scales, since it involves the oscillations at the optical frequency $1 / \epsilon$ and its harmonics, together with slow variations at the laser envelope scale.

\section{Simplification of the problem using the slowly varying envelope approximation (arbitrary laser pulse shapes)}

As what is made is conventional nonlinear optics, we can take advantage of the presence of the very different time scales. Since the laser amplitude $a(z)$ is supposed to vary slowly during one optical cycle, $\tilde{\rho}(k)$ is composed of narrow peaks at the laser wave number $1 / \epsilon$, and at its harmonics $p \times 1 / \epsilon$.

Here, we are interested by the response at low frequencies (harmonic $p=0$ ). For $\epsilon \ll 1$, we can apply the slowly varying approximation, as in the work of Stupakov and Heifets [13] to find an approximation of the integral:

$$
\begin{aligned}
\psi(k) \approx & \psi_{0}(k)=\int_{-\infty}^{+\infty} e^{-i k z} \frac{e^{-\left(z^{2} / 2\right)}}{\sqrt{2 \pi}} \\
& \times\left[\frac{1}{2 \pi} \int_{-\pi}^{+\pi} e^{i k r_{56} a(z) \sin (Z)} d Z\right] d z,
\end{aligned}
$$

which is valid when the laser pulse envelope varies slowly over one wavelength.

This gives the following approximation for the spectrum of the longitudinal charge distribution, valid in the $\mathrm{THz}$ region:

$$
\begin{aligned}
\tilde{\rho}(k) \approx & \tilde{\rho}_{0}(k) \\
= & e^{-\left[\left(r_{51}^{2}+r_{52}^{2}+r_{56}^{2}\right) / 2\right] k^{2}} \int_{-\infty}^{+\infty} e^{-i k z} \frac{e^{-\left(z^{2} / 2\right)}}{\sqrt{2 \pi}} \\
& \times J_{0}\left[k r_{56} a(z)\right] d z,
\end{aligned}
$$

with $J_{0}$ the Bessel function of zero order. This expression has to be calculated numerically in general but is far less time consuming that the original problem [Eqs. (A7) and (A9)]. Equation (A13) will be our reference relation in the following sections.

\section{Numerical calculation of typical spectra versus laser power}

Numerically, we have examined the case of a response to a Gaussian laser pulse multiplied by a sinusoidal modulation:

$$
a(z)=w_{0} e^{-\left(z^{2} / 4 \sigma_{L}^{2}\right)} \cos \left(\frac{k_{m}}{2} z+\phi\right),
$$

where $\sigma_{L}$ is the RMS width of the Gaussian in units of the bunch length $\sigma_{z}$, and $k_{m}$ is the wave number of the intensity modulation in units of $\sigma_{z}^{-1}$. $w_{0}$ represents the maximum laser-induced electron energy modulation (in the case $\phi=0$ ), in units of the relative energy spread value $\sigma_{\delta}$. The finite energy acceptance of the machine puts a strong limit on the admissible value of $w_{0}$ (and plays the role of damage threshold in conventional nonlinear optics). As an example, for UVSOR-II, the energy acceptance $\delta_{a}$ is of the order of $1 \%$, i.e., 170 times the energy spread value. Thus, we may estimate that the relevant range of values to be considered is $w_{0} \ll 170$.

As expected intuitively, numerical integration reveals that, at small values of the laser power (typically when the maximum energy modulation is such that $w_{0} \ll$ $\left.1 /\left|k_{m} r_{56}\right|\right)$, the response is mainly a single peak at $k_{m}$ [Figs. 11(a) and 11(b)]. At higher powers, deformations of the peak are predicted [Figs. 11(c) and 11(d)].

The scaling of the terahertz power versus laser power can be studied, by examining $\left|\tilde{\rho}_{0}^{2}(k)\right|$ (proportional to the terahertz pulse power spectrum) versus $w_{0}^{2}$ (since $w_{0}^{2}$ is proportional to the laser peak power). The result is displayed in Fig. 12. A quadratic dependence is observed at 

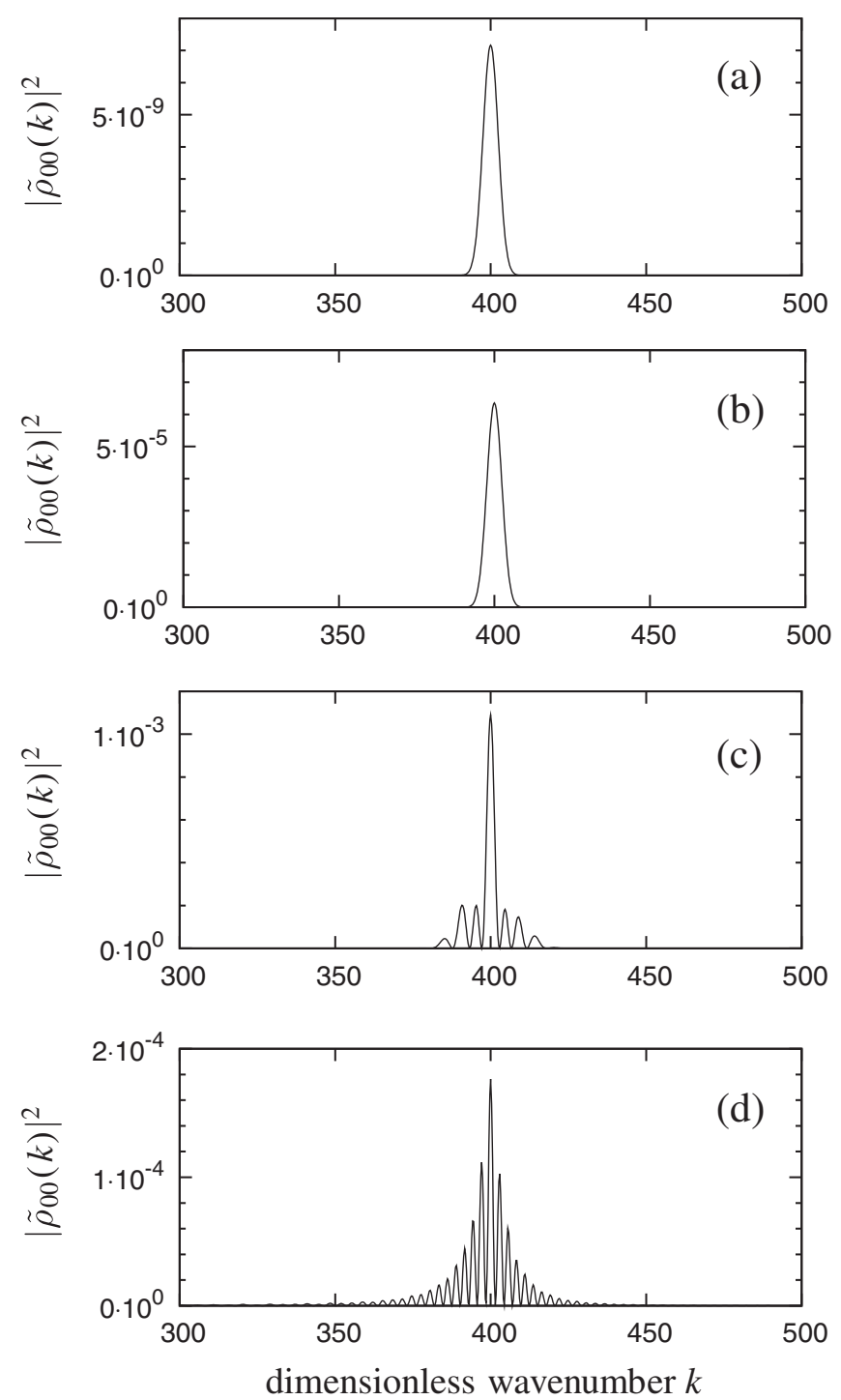

FIG. 11. Deformation of the form factor (and the emission spectrum) predicted at very high powers [numerical computation using Eq. (A13)]. (a) $w_{0}=0.1$, (b) $w_{0}=1$, (c) $w_{0}=10$, (d) $w_{0}=100$. Other parameters are $k_{m}=400, \sigma_{L}=0.3, r_{51}=$ $-0.00053, r_{52}=0.0021$, and $r_{56}=-0.0029$.

low powers, in agreement with experimental findings [see Fig. 8(b)].

Since the experimental data revealed a quadratic dependence with laser power and an absence of such spectral deformation, we can say that the conditions $w_{0} \ll$ $1 /\left|k_{m} r_{56}\right|$ were fulfilled. This motivated the perturbative analysis of the spectrum shape which will be presented in the next section. However, we cannot exclude to observe departures to the quadratic law, and related spectral deformations using higher laser powers (e.g., by operating with shorter pulses).

Finally, it is worth remarking that the domain of validity of Eq. (A13)-i.e. $\epsilon \ll 1$-is not very severe. In fact it includes most laser pulse shapes, i.e., when the amplitude

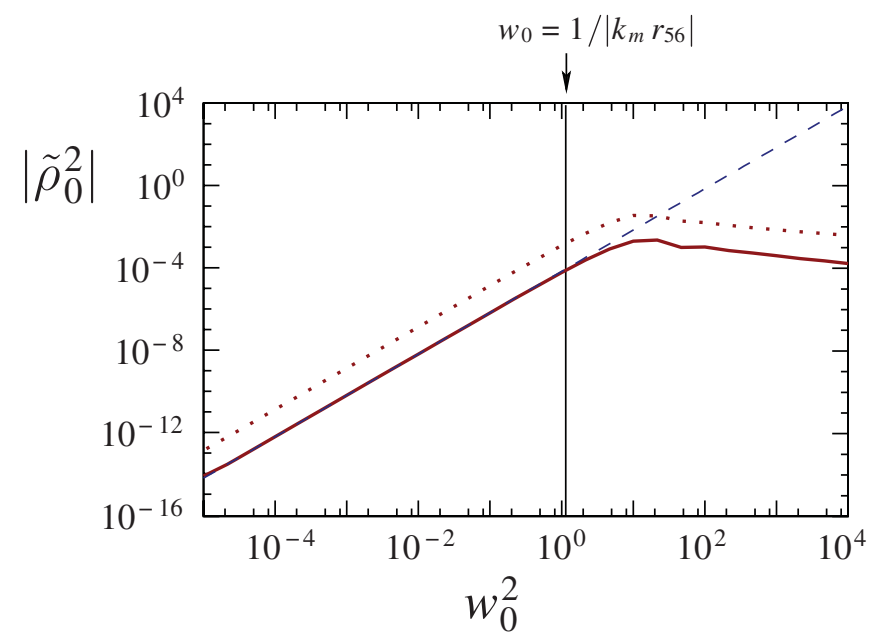

FIG. 12. (Color) Scaling of the terahertz emission power versus laser pulse power [numerical computation using Eq. (A13), and analytic results from Eq. (B16) valid when $\left.w_{0} \ll 1 /\left|k_{m} r_{56}\right|\right]$. Full and dashed lines are the maximum value of $\left|\tilde{\rho}_{0}^{2}(k)\right|$ computed numerically, and analytically, respectively (the two are indistinguishable in practice for $w_{0}<1$ here). Dotted line: the main peak area $\int\left|\tilde{\rho}_{0}^{2}(k)\right| d k$ computed numerically. Note that $w_{0}^{2}$ is proportional to the laser power, and that $\int\left|\tilde{\rho}_{0}^{2}(k)\right| d k$ is proportional to terahertz pulse energy. $\sigma_{L}=0.3, r_{51}=$ $-0.00053, r_{52}=0.0021, r_{56}=-0.0029$, and $k_{m}=400$.

varies slowly on the scale of one wavelength (typically when the pulse durations are much larger than the wavelength). In particular, Eq. (A13) is not only useful for the present problem, but can be also used for consideration of the "usual" laser slicing (i.e., with picosecond or femtosecond pulses without sine modulation) [3-6].

\section{APPENDIX B: DERIVATION OF ANALYTIC EXPRESSIONS OF THE SPECTRUM IN THE LIMIT OF LOW LASER POWER}

Equation (A13) requires numerical calculation of an integral. However, analytical expressions can be obtained in the limit of small laser powers.

\section{Asymptotic expansion valid for small laser intensities and arbitrary laser pulse shape}

We now examine the limit where $a(z)$ is small. In the regions of the spectrum where $|a(z)| \ll 1 /\left|k r_{56}\right|$, we can expand Eq. (A13) to second order:

$$
\begin{aligned}
\tilde{\rho}_{0}(k) \approx & \tilde{\rho}_{00}(k) \\
= & e^{-\left[\left(r_{51}^{2}+r_{52}^{2}+r_{56}^{2}\right) / 2\right] k^{2}} \int_{-\infty}^{+\infty} e^{-i k z} \frac{e^{-\left(z^{2} / 2\right)}}{\sqrt{2 \pi}} \\
& \times\left[1-\frac{k^{2} r_{56}^{2}}{4} a^{2}(z)\right] d z .
\end{aligned}
$$

This leads to 


$$
\begin{aligned}
\tilde{\rho}_{00}(k)= & e^{-\left[\left(r_{51}^{2}+r_{52}^{2}+r_{56}^{2}\right) / 2\right] k^{2}}\left(e^{-\left(k^{2} / 2\right)}-\frac{k^{2} r_{56}^{2}}{4}\right. \\
& \left.\times \int_{-\infty}^{+\infty} e^{-i k z} \frac{e^{-\left(z^{2} / 2\right)}}{\sqrt{2 \pi}} a^{2}(z) d z\right) .
\end{aligned}
$$

This basic Taylor expansion is valid for any pulse shape, and may be used for obtaining analytical expressions, in particular, cases. In the next sections, we present the case of an infinitely long laser pulse and electron bunch, and then the case of a Gaussian laser pulse multiplied by a sinusoidal modulation. Similar calculations may be performed at will for other laser pulse shapes.

\section{Analytical expression in the limit of infinitely long bunch and laser pulse}

At small laser power, we can perform an asymptotic expansion, leading to simple analytical expressions. In the limit of infinitely long bunch and laser pulse, if we replace $\frac{e^{-\left(z^{2} / 2\right)}}{\sqrt{2 \pi}}$ by 1 , and take $a(z)=\sqrt{P} \cos k_{m} z / 2, \tilde{\rho}_{00}(k)$ becomes

$$
\begin{aligned}
\tilde{\rho}_{00 \infty}(k)= & e^{-\left[\left(r_{51}^{2}+r_{52}^{2}+r_{56}^{2}\right) / 2\right] k^{2}} \\
& \times \int_{-\infty}^{+\infty} e^{-i k z}\left[1-\frac{k^{2} r_{56}^{2}}{4} P \cos ^{2}\left(\frac{k_{m}}{2} z\right)\right] d z \\
= & 2 \pi \delta(k)-e^{-\left[\left(r_{51}^{2}+r_{52}^{2}+r_{56}^{2}\right) / 2\right] k^{2}}\left(\frac{k^{2} r_{56}^{2} P \pi}{8}\right) \\
& \times\left[\delta\left(-k+k_{m}\right)+\delta\left(k+k_{m}\right)\right] .
\end{aligned}
$$

The first term is the contribution which we would have if the electron bunch were unmodulated. The second one is the contribution due to the laser modulation. We thus expect that, in first approximation, the $\mathrm{THz}$ power depends on the excitation frequency $k_{m}$ with the following law:

$$
P_{T H z} \approx C r_{56}^{4} P^{2} k^{4} e^{-\left(r_{51}^{2}+r_{52}^{2}+r_{56}^{2}\right) k^{2}},
$$

where $C$ is an unknown parameter at this level of modeling.

This curve has a maximum located at

$$
k_{m}=k_{\text {mopt }}=\frac{\sqrt{2}}{\sqrt{r_{51}^{2}+r_{52}^{2}+r_{56}^{2}}} .
$$

In terms of the dimensioned variables, the associated frequency (in hertz) is

$$
\nu_{\text {mopt }}=\frac{c}{2 \pi} \frac{\sqrt{2}}{\sqrt{R_{51}^{2} \sigma_{x}^{2}+R_{52}^{2} \sigma_{x^{\prime}}^{2}+R_{56}^{2} \sigma_{\delta}^{2}}} .
$$

This analytical value can be used to estimate the best efficiency region with a good approximation (i) when the laser pulse is much longer than the modulation period, and (ii) in the limit of low laser powers. The validity of the results is confirmed by the good agreement with experimental data (see Sec. IV).

\section{Small intensities, modulation with slowly varying envelope}

Similar calculations can be used to get asymptotic approximations considering more realistic laser pulses shapes, and a Gaussian electron bunch. As an example, analytical expressions in the case of finite-size Gaussian electron beam and a Gaussian laser pulse [defined by Eq. (A14)] will be derived below. However, it is worth considering first a more general form for the laser pulse:

$$
a(z)=A(z) \times \sqrt{2} \cos \left(\frac{k_{m}}{2} z+\phi\right),
$$

in the limit $k_{m} \gg 1$, and with $A(z)$ slowly varying with respect to the sinusoidal modulation. More precisely, the Fourier transform of $A$ has noticeable values only when $|k| \ll k_{m}$. This includes the case of a laser pulse defined by Eq. (A14), in which case $A(z)=w_{0} e^{-\left(z^{2} / 4 \sigma_{L}^{2}\right)} / \sqrt{2}$. We obtain easily

$$
\begin{aligned}
& \psi_{01}(k)=-\frac{k^{2} r_{56}^{2}}{4}\left(F(k)+\frac{1}{2} F\left(k-k_{m}\right) e^{2 i \phi}\right. \\
&\left.+\frac{1}{2} F\left(k+k_{m}\right) e^{-2 i \phi}\right) \\
& \text { with }: F(k)= \int_{-\infty}^{+\infty} e^{-i k z}\left[\frac{e^{-\left(z^{2} / 2\right)}}{\sqrt{2 \pi}} A^{2}(z)\right] d z \\
& \tilde{\rho}_{0}(k) \approx \tilde{\rho}_{00}(k) \\
& \approx e^{-\left[\left(r_{51}^{2}+r_{52}^{2}+r_{56}^{2}\right) / 2\right] k^{2}}\left[e^{-k^{2} / 2}-\frac{k^{2} r_{56}^{2}}{4} F(k)\right. \\
&-\frac{1}{2} \frac{k^{2} r_{56}^{2}}{4} F\left(k-k_{m}\right) e^{2 i \phi} \\
&\left.-\frac{1}{2} \frac{k^{2} r_{56}^{2}}{4} F\left(k+k_{m}\right) e^{-2 i \phi}\right] .
\end{aligned}
$$

The four terms correspond to precise physical effects: (a) $e^{-\left[\left(r_{56}^{2}+r_{51}^{2}+r_{52}^{2}\right) / 2\right] k^{2}} \times e^{-\left(k^{2} / 2\right)}$ is the contribution of the electron bunch without laser interaction; (b) $e^{-\left[\left(r_{56}^{2}+r_{51}^{2}+r_{52}^{2}\right) / 2\right] k^{2}} \times\left[-\frac{k^{2} r_{56}^{2}}{4} F(k)\right]$ is the contribution which would have a Gaussian laser pulse with same energy, but without modulation ("traditional slicing"); (c) $e^{-\left[\left(r_{56}^{2}+r_{51}^{2}+r_{52}^{2}\right) / 2\right] k^{2}} \times\left[-\frac{1}{2} \frac{k^{2} r_{56}^{2}}{4} F\left(k \pm k_{m}\right) e^{\mp 2 i \phi}\right]$ are the terms due to the modulation, and which are of interest here. It is strongly peaked for $k$ near $\pm k_{m}$. In these regions, the other terms are negligible.

In the limit of low power, we have [see Eq. (B1)]

$$
\tilde{\rho}_{0}(k) \approx \tilde{\rho}_{00}(k)=e^{-\left[\left(r_{56}^{2}+r_{51}^{2}+r_{52}^{2}\right) / 2\right] k^{2}}\left[\psi_{00}(k)+\psi_{01}(k)\right]
$$

with

$$
\psi_{00}(k)=e^{-\left(k^{2} / 2\right)}
$$




$$
\psi_{01}(k)=-\frac{k^{2} r_{56}^{2}}{4} \int_{-\infty}^{+\infty} e^{-i k z} \frac{e^{-\left(z^{2} / 2\right)}}{\sqrt{2 \pi}} a^{2}(z) d z .
$$

The first term which is just the CSR emission of the unperturbed electron bunch will be neglected later. Technically, we just have to evaluate $\psi_{01}(k)$ :

$$
\begin{aligned}
\psi_{01}(k)= & -\frac{k^{2} r_{56}^{2}}{4} \int_{-\infty}^{+\infty} e^{-i k z}\left[\frac{e^{-\left(z^{2} / 2\right)}}{\sqrt{2 \pi}} A^{2}(z)\right] \\
& \times\left[1+\cos \left(k_{m} z+2 \phi\right)\right] d z .
\end{aligned}
$$

\section{Particular case of a sinusoidal modulation with Gaussian envelope, and low laser power}

This type of approach can be used to derive asymptotic approximations in various cases. If we consider a laser pulse with sinusoidal modulation and Gaussian shape,

$$
a(z)=w_{0} e^{-\left(z^{2} / 4 \sigma_{L}^{2}\right)} \times \cos \left(\frac{k_{m}}{2} z+\phi\right),
$$

i.e., $A(z)=w_{0} e^{-\left(z^{2} / 4 \sigma_{L}^{2}\right)} / \sqrt{2}$, we have

$$
-\frac{1}{2} \frac{r_{56}^{2} k^{2}}{4} F(k)=-\frac{w_{0}^{2} k_{m}^{2} r_{56}^{2} \sigma_{L}}{32 \sqrt{\sigma_{L}^{2}+1}} e^{-\left[\sigma_{L}^{2}\left(k-k_{m}\right)^{2} / 2\left(\sigma_{L}^{2}+1\right)\right]} .
$$

Neglecting the (a) and (b) contributions, and the overlap between the two peaked functions in (c), we obtain the following approximation for the form factor:

$$
\left|\tilde{\rho}_{0}(k)\right|^{2} \approx e^{-\left(r_{56}^{2}+r_{51}^{2}+r_{55}^{2}\right) k^{2}} \frac{w_{0}^{4} k_{m}^{4} r_{56}^{4} \sigma_{L}^{2}}{256\left(\sigma_{L}^{2}+1\right)} e^{-\left[\sigma_{L}^{2}\left(k-k_{m}\right)^{2} /\left(\sigma_{L}^{2}+1\right)\right]} .
$$

Two tests of this approximation versus numerical computation are presented in Figs. 12 and 13. More generally,

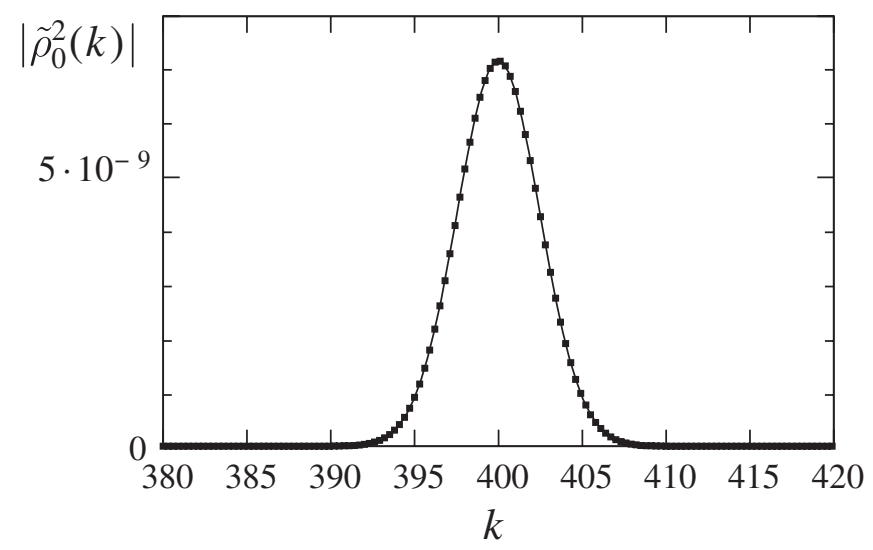

FIG. 13. Form factor in the case of a Gaussian laser pulse with internal modulation: Numerical result from Eq. (A13) (dots) and approximation from Eq. (B16) (full line). $k_{m}=400, \sigma_{L}=0.3$, $w_{0}=0.1, r_{51}=-0.00053, r_{52}=0.0021$, and $r_{56}=-0.0029$. numerical tests confirm the fact that Eq. (B16) is valid when the conditions $\sigma_{L} \gg 1 / k_{m}$ and $|a(z)| \ll 1 /\left|k_{m} r_{56}\right|$.

If we neglect the contribution of the $e^{-\left[\left(r_{56}^{2}+r_{51}^{2}+r_{52}^{2}\right) / 2\right] k^{2}}$ term, the RMS width of the peak is

$$
\Delta k_{\mathrm{RMS}}=\frac{1}{\sqrt{2}} \sqrt{1+\frac{1}{\sigma_{L}^{2}}} .
$$

In terms of the physical variables the RMS width of the peak can be written (in hertz)

$$
\begin{gathered}
\Delta \nu_{\mathrm{RMS}}=\frac{c \Delta k_{\mathrm{RMS}}}{2 \pi \sigma_{z}} \\
=\frac{1}{2 \pi \sqrt{2}} \sqrt{\frac{1}{\Delta T_{\mathrm{LRMS}}^{2}}+\frac{1}{\Delta T_{\mathrm{zRMS}}^{2}}},
\end{gathered}
$$

where $\Delta T_{\mathrm{LRMS}}$ and $\Delta T_{\mathrm{zRMS}}$ are the values of the RMS durations of the laser pulse and the electron bunch, respectively (in seconds).

However, it is important to note that we supposed here the ideal case of sine modulations without any chirp in the modulation frequency. For the experiments presented in the present paper, the parameters are $\Delta T_{\text {LRMS }}=130 \mathrm{ps}$ and $\Delta T_{\text {zRMS }}=100 \mathrm{ps}$. Hence, in the absence of chirp, we could have theoretically $\Delta \nu_{\mathrm{RMS}}=0.07 \mathrm{~cm}^{-1}$. The values measured experiment are larger: $\approx 0.14 \mathrm{~cm}^{-1}$ and $0.38 \mathrm{~cm}^{-1}$ for the peaks (1) and (0), respectively, these values depending on the selected frequency [in contrast to what is predicted by Eq. (B19)]. This can be attributed to the existence of a chirp in the laser pulse internal modulation, an effect which is well known in the framework of narrowband terahertz emission in semiconductors [15]. Calculation of this effect is left for studies. Such calculation will require the knowledge of the details of stretched pulse at the output of the amplifier (or equivalently the third-order dispersion of the output grating), but the calculation of spectra using these data is in principle similar to the examples presented here. Beside this, we think also that attempting to reduce the nonlinear chirp at the amplifier's output will have a drastic effect on the linewidth.

\section{APPENDIX C: EFFECT OF DISPERSION IN THE INITIAL ELECTRON BUNCH DISTRIBUTION}

The present calculation can be extended to include a finer description of the initial electron bunch distribution. In this Appendix, we include the effect of finite dispersion. We consider an initial bunch distribution of the type

$$
f\left(x, x^{\prime}, z, w\right)=\frac{1}{4 \pi^{2}} e^{-(1 / 2)\left[(x-\eta w)^{2}+x^{\prime 2}+z^{2}+w^{2}\right]},
$$

where $\eta$ characterizes the transverse energy dispersion. After interaction with the laser, 


$$
g\left(x, x^{\prime}, z, w\right)=\frac{1}{4 \pi^{2}} e^{-(1 / 2)\left\{(x-\eta w)^{2}+x^{\prime 2}+z^{2}+[w-a(z) \sin (z / \epsilon)]^{2}\right\}} .
$$

Using Eq. (D4), we obtain

$$
\begin{aligned}
\tilde{\rho}(k)= & \int_{-\infty}^{+\infty} \int_{-\infty}^{+\infty} \int_{-\infty}^{+\infty} \int_{-\infty}^{+\infty} d x d x^{\prime} d z d w \\
& \times e^{-i k\left(r_{51} x_{1}+r_{52} x_{1}^{\prime}+z+r_{56} w_{1}\right)} \frac{1}{4 \pi^{2}} \\
& \times e^{-(1 / 2)\left\{(x-\eta w)^{2}+x^{\prime 2}+z^{2}+[w-a(z) \sin (z / \epsilon)]^{2}\right\}} .
\end{aligned}
$$

Quadratures over $x, x^{\prime}, e$, and slowly varying laser envelope approximation can then be applied in a similar way as in the previous sections. The spectrum of the longitudinal distribution [Eq. (A13)] is transformed into

$$
\begin{aligned}
\tilde{\rho}(k) \approx & \tilde{\rho}_{0}(k) \\
= & e^{-\left[r_{51}^{2}+r_{52}^{2}+\left(r_{51} \eta+r_{56}\right)^{2}\right] k^{2} / 2} \int_{-\infty}^{+\infty} e^{-i k z} \frac{e^{-\left(z^{2} / 2\right)}}{\sqrt{2 \pi}} \\
& \times J_{0}\left[k r_{56} a(z)\right] d z .
\end{aligned}
$$

In the low power approximation, the frequency at the response maximum (B4) becomes

$$
k_{m}=k_{\mathrm{mopt}}=\frac{\sqrt{2}}{\sqrt{r_{51}^{2}+r_{52}^{2}+\left(r_{51} \eta+r_{56}\right)^{2}}},
$$

and more generally each result of the previous sections can be extended to the $\eta \neq 0$ case, just by replacing $r_{56}$ by $\left(r_{51} \eta+r_{56}\right)$.

\section{APPENDIX D: DETAIL OF THE CHANGE OF VARIABLES USED FOR THE INTEGRATION}

To obtain Eq. (A10), we proceeded to a change of variables. Substituting Eq. (A7) in Eq. (A8) leads to

$$
\begin{aligned}
\tilde{\rho}(k)= & \int_{-\infty}^{+\infty} \int_{-\infty}^{+\infty} \int_{-\infty}^{+\infty} \int_{-\infty}^{+\infty} d x d x^{\prime} d z d w e^{-i k z} \\
& \times h\left(x, x^{\prime}, z, w\right) .
\end{aligned}
$$

Then, using the relation (A6), this expression may be expressed as

$$
\begin{aligned}
\tilde{\rho}(k)= & \int_{-\infty}^{+\infty} \int_{-\infty}^{+\infty} \int_{-\infty}^{+\infty} \int_{-\infty}^{+\infty} d x d x^{\prime} d z d w e^{-i k z}|\mathbf{r}|^{-1} \\
& \times g\left[\mathbf{r}^{-1}\left(x, x^{\prime}, z, w\right)\right] .
\end{aligned}
$$

Note that in our case $|\mathbf{r}|=1$; however, we will not use this property in the following. Now we proceed to the change of variables:

$$
\left(\begin{array}{c}
x_{1} \\
x_{1}^{\prime} \\
z_{1} \\
w_{1}
\end{array}\right)=\mathbf{r}^{-1}\left(\begin{array}{c}
x \\
x^{\prime} \\
z \\
w
\end{array}\right)
$$

Since we have $d x d x^{\prime} d z d w=|\mathbf{r}| d x_{1} d x_{1}^{\prime} d z_{1} d w_{1}$, we obtain

$$
\begin{aligned}
\tilde{\rho}(k)= & \int_{-\infty}^{+\infty} \int_{-\infty}^{+\infty} \int_{-\infty}^{+\infty} \int_{-\infty}^{+\infty} d x_{1} d x_{1}^{\prime} d z_{1} d w_{1} \\
& \times e^{-i k\left(r_{51} x_{1}+r_{52} x_{1}^{\prime}+z+r_{56} w_{1}\right)} g\left(x_{1}, x_{1}^{\prime}, z_{1}, w_{1}\right) .
\end{aligned}
$$

Then after calculating the quadratures over $x_{1}, x_{1}^{\prime}$, and $w_{1}$, Eq. (A10) is easily obtained. Note that the result depends only on the $r_{5 i}$ matrix elements and the electron bunch state just after interaction with the laser.

[1] R. W. Schoenlein, S. Chattopadhyay, H. H. W. Chong, T. E. Glover, P. A. Heimann, W. P. Leemans, C. V. Shank, A. Zholents, and M. Zolotorev, Appl. Phys. B 71, 1 (2000).

[2] R. W. Schoenlein, S. Chattopadhyay, H. H. W. Chong, T. E. Glover, P. A. Heimann, C. V. Shank, A. A. Zholents, and M. S. Zolotorev, Science 287, 2237 (2000).

[3] S. Khan, K. Holldack, T. Kachel, R. Mitzner, and T. Quast, Phys. Rev. Lett. 97, 074801 (2006).

[4] J. M. Byrd, Z. Hao, M. C. Martin, D. S. Robin, F. Sannibale, R. W. Schoenlein, A. A. Zholents, and M. S. Zolotorev, Phys. Rev. Lett. 96, 164801 (2006).

[5] K. Holldack, T. Kachel, S. Khan, R. Mitzner, and T. Quast, Phys. Rev. ST Accel. Beams 8, 040704 (2005).

[6] M. Shimada, M. Katoh, S. Kimura, A. Mochihashi, M. Hosaka, Y. Takashima, T. Hara, and T. Takahashi, Jpn. J. Appl. Phys. 46, 7939 (2007).

[7] Y. Takashima, M. Katoh, M. Hosaka, and A. Mochihashi, UVSOR Activity Report 2002, No. UVSOR-30, 2003 pp. 56-77.

[8] S. Bielawski, C. Evain, T. Hara, M. Hosaka, M. Katoh, S. Kimura, A. Mochihashi, M. Shimada, C. Szwaj, T. Takahashi, and Y. Takashima, Nature Phys. 4, 390 (2008).

[9] G. P. Williams, Nature Phys. 4, 356 (2008).

[10] F. Sannibale, A. Marcelli, and P. Innocenzi, J. Synchrotron Radiat. 15, 655 (2008).

[11] J. G. Neumann, R. B. Fiorito, P. G. O'Shea, H. Loos, B. Sheehy, Y. Shen, and Z. Wu, J. Appl. Phys. 105, 053304 (2009).

[12] D. Xiang and G. Stupakov, Phys. Rev. ST Accel. Beams 12, 080701 (2009).

[13] G. Stupakov and S. Heifets, in Proceedings of the 10th European Particle Accelerator Conference, Edinburgh, Scotland, 2006 (EPS-AG, Edinburgh, Scotland, 2006), p. 3502, THPLS097.

[14] S. Kimura, E. Nakamura, T. Nishi, Y. Sakurai, K. Hayashi, J. Yamazaki, and M. Katoh, Infrared Phys. Technol. 49, 147 (2006).

[15] A. S. Weling and D. H. Auston, J. Opt. Soc. Am. B 13, 2783 (1996).

[16] Y. Takagi, T. Kobayashi, K. Yoshihara, and S. Imamura, Opt. Lett. 17, 658 (1992).

[17] J. Ranka, A. L. Gaeta, A. Baltuska, M.S. Pshenichnikov, and D. A. Wiermsa, Opt. Lett. 22, 1344 (1997).

[18] L. I. Schiff, Rev. Sci. Instrum. 17, 6 (1946).

[19] J. Schwinger, Phys. Rev. 75, 1912 (1949).

[20] J. S. Nodvick and D. S. Saxon, Phys. Rev. 96, 180 (1954). 
[21] C. J. Hirschmugl, M. Sagurton, and G. P. Williams, Phys. Rev. A 44, 1316 (1991).

[22] G. L. Carr, M. C. Martin, W. R. McKinney, K. Jordan, G. Neil, and G. Williams, Nature (London) 420, 153 (2002).

[23] K. Holldack, T. Kachel, S. Khan, R. Mitzner, and T. Quast, Phys. Rev. ST Accel. Beams 8, 040704 (2005).

[24] Y.R. Shen, The Principles of Nonlinear Optics (Wiley Classics Library, New York, 2002).
[25] M. Bass, P. A. Franken, J.F. Ward, and G. Weinreich, Phys. Rev. Lett. 9, 446 (1962).

[26] A. Gover, Phys. Rev. ST Accel. Beams 8, 030701 (2005).

[27] A. Gover, E. Dyunin, Y. Lurie, Y. Pinhasi, and M. V. Krongauz, Phys. Rev. ST Accel. Beams 8, 030702 (2005).

[28] J. M. Byrd, Z. Hao, M. C. Martin, D. S. Robin, F. Sannibale, R. W. Schoenlein, A. A. Zholents, and M. S. Zolotorev, Phys. Rev. Lett. 97, 074802 (2006). 